\title{
Spectral Narrowing in Coherent Rayleigh Scattering
}

\author{
H. T. Bookey \\ Physics, School of Engineering and Physical Sciences, Heriot-Watt University, Edinburgh, Scotland EH14 4AS, United Kingdom
}

M. N. Shneider

Department of Mechanical and Aerospace Engineering, Princeton University, Princeton, New Jersey 08544, USA

P. F. Barker

Department of Physics and Astronomy, University College London, WClE 6BT, United Kingdom

(Received 9 May 2007; published 26 September 2007)

Spectral narrowing of the coherent Rayleigh scattering line shape in a room temperature $\mathrm{CO}_{2}$ gas $\left(2.5 \times 10^{23} \mathrm{~m}^{-3}\right)$ with intense fields in the $10^{15} \mathrm{~W} \mathrm{~m}^{-2}$ range is observed. The line shape saturates to a width of approximately half that observed at low pump intensities and indicates a transition from scattering primarily from untrapped molecules to that from both trapped and untrapped molecules that are localized by the deep $(60 \mathrm{~K})$ optical potentials produced by the pump beams. At higher densities $\left(5 \times 10^{24} \mathrm{~m}^{-3}\right)$, collisions between the trapped and untrapped molecules broaden the spectral profile.

In atomic and molecular gases the finite momentum spread of an ensemble of particles leads to the broadening of spectral profiles due to the Doppler effect. One important interaction that leads to the narrowing of spectral features occurs when the particles are confined to a region comparable to or smaller than the wavelength of light used to excite the species. This effect was first considered by Dicke [1], but was also observed by Mössbauer in the absorption spectrum of gamma radiation in the crystal structure of a solid due to the confinement of the atomic species [2]. Dicke narrowing is commonly observed in spectral profiles in high-density gases [3], but in more recent years this effect has also been studied in the spectra of ultracold ions and neutral atoms that are tightly confined in well-defined potentials created by an externally applied field $[4,5]$. These spectral features are particularly apparent within the spatially periodic optical traps of an optical lattice which has dimensions on the scale of an optical wavelength [6]. Because of the periodic nature of the trapped atoms in an optical lattice, phase matched scattering [7] can be used to probe long range order [8] and atomic dynamics [9] within the trap. In this Letter, we describe the first observation of Bragg scattering or coherent Rayleigh scattering from a relatively "hot", room temperature gas which is trapped by deep optical lattice potentials, utilizing a far off-resonant pulsed optical field. In our experiments we observe a spectral narrowing in a coherent scattering process that arises due to the confinement and trapping of room temperature particles in deep optical lattices but in our case is not attributed to the Dicke or Mössbauer effect.

Coherent Rayleigh scattering is an optical, four-wave mixing technique [10] that has been used to determine temperature and gas composition from the scattered line shape. This nonresonant four-wave mixing process, which is similar to other nonresonant grating based techniques $[11,12]$, uses two crossed pump beams $I_{1}$ and $I_{2}$ as shown in Fig. 1, to create a density grating in the sample via a periodic optical dipole force. The dipole force perturbs the motion of the molecules that are travelling near to the speed of the interference pattern of the crossed pump fields. By tuning the frequency difference between the two pump fields a perturbation centered at a particular velocity within the Doppler profile is created. The relative magnitude of the induced density perturbation at each velocity or frequency difference, can be determined by measurement of the relative intensity of a third Bragg scattered probe field, $I_{3}$, as shown in Fig. 1. For nondepleted pump fields, the intensity of the Bragg scattered field is $I_{s} \sim \delta \rho^{2} I_{1} I_{2} I_{3}$ where $\delta \rho$ is the periodic density perturbation induced by the two pump beams. The relative strength of $\delta \rho$ at different pump field detunings determines the strength of scattered signal field and ultimately the

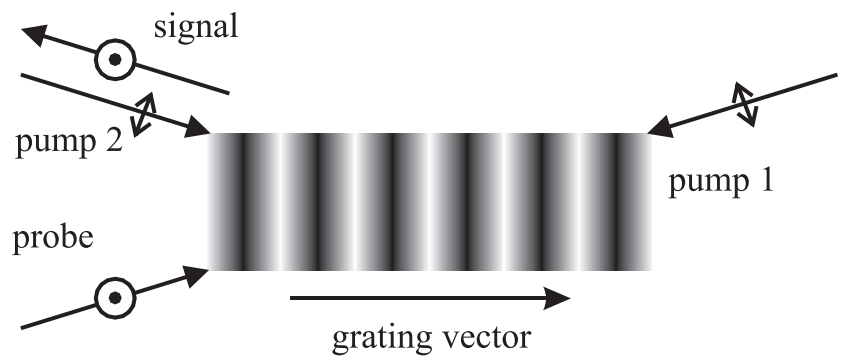

FIG. 1. A diagram illustrating the nonresonant four-wave mixing process that forms the coherent Rayleigh scattering signal. Pump beam 1 and 2 form a density perturbation in the gas via the dipole force and a weaker probe beam is Bragg scattered from the density perturbation to create the signal beam which is recorded as a function of the detuning between the two pump beams. 
coherent Rayleigh scattering (CRS) line shape. The magnitude of this density perturbation is controlled by the depth of the optical potential induced by the two pump beams along the $x$ axis and is given by $U(x, t)=$ $\frac{2 \alpha I}{c \varepsilon_{0}} \cos ^{2}(q x-\Omega t)$, where $\alpha$ is the polarizability of gas, $I(t)=\sqrt{I_{1} I_{2}} ; I_{1}, I_{2}$ are the intensities of each pump beam, $q$ is the wave vector of the interference pattern and $\Omega$ is the frequency difference between the two pump beams. The perturbation at each pump frequency is determined by solving the Boltzmann equation subject to the external periodic force of the optical potential $(F=-\nabla U)$ [13]. The square of the induced density at each velocity is given by $\delta \rho^{2}=\rho^{2} \int_{0}^{2 \pi / q}\left|\int_{-\infty}^{\infty} \delta f(x, v, t) d v\right|^{2} d x$, where $\delta f(x, v, t)$ is the optically induced periodic perturbation to the equilibrium velocity distribution function. For low intensities $\left(<10^{14} \mathrm{~W} \mathrm{~m}^{-2}\right)$, where $U / k_{b} \ll T$, the total perturbation, $\delta \rho$, to the velocity distribution function of the gas is small and nonintrusive measurements of the gas temperature can be made [14,15]. At low intensities in the collisionless regime, an analytical solution to $\delta f$ has been found and the spectral profile is well approximated by a Gaussian function that is approximately $10 \%$ wider than the spontaneous Rayleigh scattering line shape [10]. Collisions affect this line shape at higher densities when the mean free path is comparable to the grating period. In this regime the line shape is broadened and dominated by Brillouin features [16]. In this Letter we report on the observation of line narrowing in CRS line shapes in the high intensity regime where the periodic optical potential created by the pump beams is large enough so that unlike conventional CRS, trapped molecules become the major contributor to the CRS signal [17]. We explore primarily the collisionless regime, but also consider scattering at higher densities in the kinetic regime where collisions affect this process.

To understand how changes in the CRS line shape occur with an increase in pump intensity, it is useful to consider how the velocity distribution function of the gas changes with intensity. Figure 2 contains plots of the 1D distribution function of $\mathrm{CO}_{2}$ gas at $295 \mathrm{~K}(\alpha=2.88 \times$ $\left.10^{-40} \mathrm{C} \mathrm{m}^{2} / \mathrm{V}\right)$ in the $(x, v)$ plane where the $x$ direction is along the axis of the counterpropagating pump beams and $v$ is the velocity along this axis. The color in the graph indicates relative density. Figure 2(a) is the distribution function in the absence of the potential and Figs. 2(b) and 2(c) are for two optical potentials of well depth 3.9 and $62 \mathrm{~K}$. The perturbed distribution function is calculated by numerical integration of the collisionless Boltzmann equation. Figure 2(b) shows the distribution function modified by the weakest optical potential $U / k_{b}=3.9 \mathrm{~K} \ll T$ $\left(2.5 \times 10^{14} \mathrm{~W} / \mathrm{m}^{2}\right)$ traveling at a velocity of $236 \mathrm{~m} \mathrm{~s}^{-1}$. Also shown on this graph are two lines equidistant around the velocity of the potential which represents the maximum and minimum range in velocity that trapped particles within the potential can attain, given by $\Delta v=\sqrt{\frac{4 a I}{c \varepsilon_{0} M}}$.
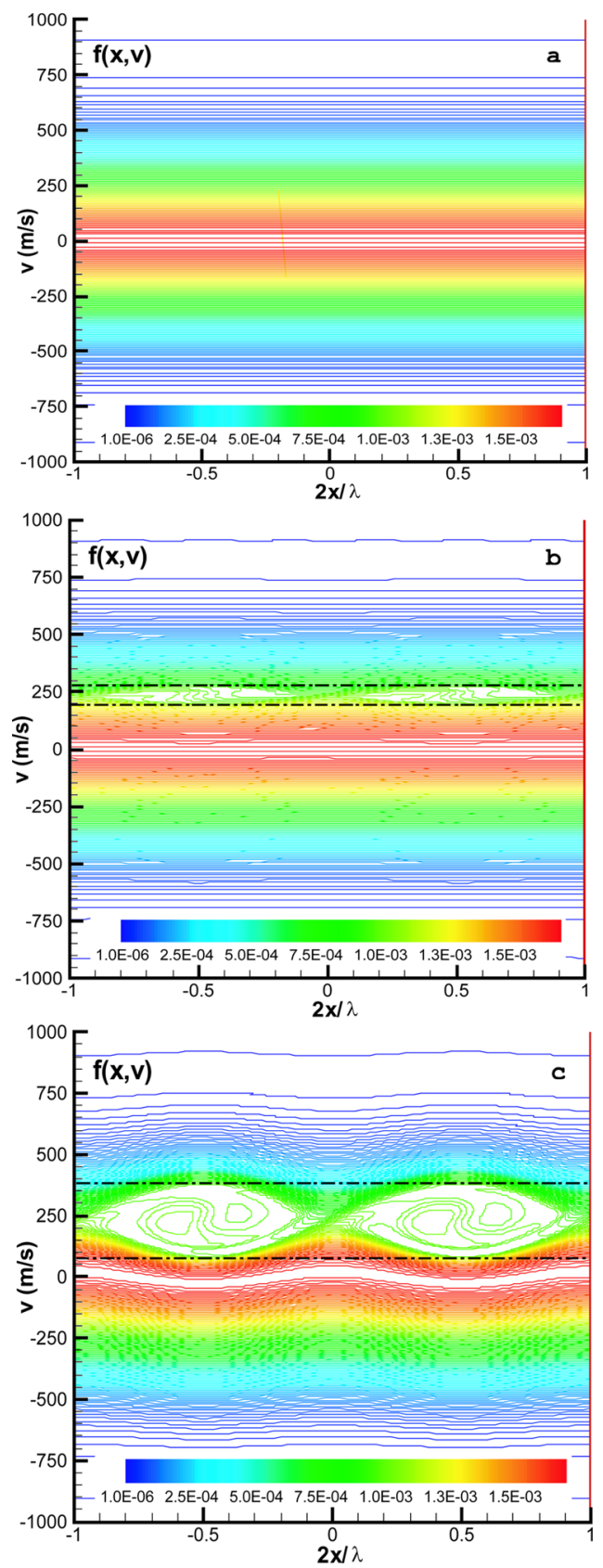

FIG. 2 (color online). Calculated 1D velocity distribution functions for $\mathrm{CO}_{2}$ gas subject to the periodic potential of the pump fields $(T=295 \mathrm{~K})$ in, (a) the absence of the field and, (b) when a weak optical potential of well depth $(3.9 \mathrm{~K})$ is applied to the gas. Graph (c) is the perturbation for a larger potential $(62 \mathrm{~K})$ where the perturbation is more localized within the trapped region.

Note that this periodic perturbation along the $x$ axis leads to the coherent scattering signal. This relatively weak perturbation extends significantly beyond the bounds of the trapped region in the $v$ axis of the distribution function and accounts for the broadened CRS spectral profile which is $10 \%$ wider than the spontaneous scattered profile. This occurs because even when the velocity of the optical lattice is detuned into the wings of the velocity distribution func- 
tion (i.e., when the velocity of the potential exceeds the $236 \mathrm{~m} / \mathrm{s}$ values shown in this figure), perturbations from near the center of the distribution, where the density is high, contribute significantly to $\delta \rho^{2}$ and thus the scattered signal even when they are not trapped. Figure 2(c) is the calculated perturbation to the distribution function for a higher intensity case in which $U / k_{b}=62 \mathrm{~K} \sim T(4.0 \times$ $10^{15} \mathrm{~W} / \mathrm{m}^{2}$ ). The two horizontal lines represent the maximum extent of molecules trapped by the potential which is now clearly visible as the elliptical regions with closed contours centered at the velocity of the potential. The trapped molecules in this region are forced to oscillate within the potential and this motion creates a plateau within the distribution function at a density that is approximately equal to the unperturbed density at the velocity of the optical potential. Like Fig. 2(b), the whole distribution function is perturbed by the potential, but now the largest perturbation is produced within closer proximity to the trapped region. This group of molecules within a smaller velocity spread will dominate the scattering process at this velocity. As the value of the average distribution function in this plateau region is approximately equal to that of the original distribution function we can expect that at this, and similar velocities where a significant fraction of the distribution is trapped, the CRS signal will be approximately proportional to the square of the original distribution function which is less than half the width of the CRS signal at low intensity. Our calculations for larger well depths show that even when a wider plateau is created the average value of the perturbation in the trapped region is still approximately equal to the unperturbed distribution function and thus we expect that the spectral profile will have a similar width over a wide range of potential well depths where most of the perturbation comes from trapped particles. Figure 2(c) also shows that a significant fraction of molecules are never trapped by the potential because they are at an initial velocity and/or phase with respect to the potential where they cannot be trapped. This untrapped fraction increases as the velocity of the optical potential is increased and we should expect that the profile in the wings should be similar to that observed at low intensity where the majority of the perturbed distribution function is not trapped by the potential.

To measure the line shape at high intensity we employ narrow bandwidth CRS scattering [15,18]. To produce the three beams used for CRS, the output from a frequency doubled $Q$-switched Nd:YAG laser operating at $532 \mathrm{~nm}$ (100 MHz bandwidth) was split into two beams to form pump beam 1 and the probe beam. A narrow-band $(100 \mathrm{MHz}) \mathrm{CW}$ Nd:YAG laser was pulse amplified and frequency doubled to produce pump beam 2 . All beams had pulse durations of $\sim 10 \mathrm{~ns}$ and approximately Gaussian spatial and temporal profiles. The probe beam polarization was orthogonal to that of the two pump beams which were focused into a gas cell by two $200 \mathrm{~mm}$ focal length lenses producing an interaction length of approximately $10 \mathrm{~mm}$. The gas cell was $300 \mathrm{~mm}$ long and had antireflection coated windows. The probe beam counter-propagated to the path of pump 1 and the resulting CRS signal counterpropagated to pump beam 2 . The signal polarization was orthogonal to the pump beam polarization and was extracted by a polarizer which was then passed through another polarizer to remove scattered light and focused onto a silicon photodiode. The frequency of pump beam 1 and the probe was scanned and the frequency difference was determined by measurement of the beat frequency of the fundamental radiation on a fast InGaAS photodiode. CRS spectra were recorded by scanning the frequency difference between the two pump lasers and measuring the relative intensity of the scattered probe light. All of these signals were integrated over $10-100$ shots on a boxcar averager at each frequency difference.

CRS spectra were recorded for $\mathrm{CO}_{2}$ gas at room temperature $(295 \mathrm{~K})$ and at pressures of less than $200 \mathrm{mbar}$ over a range of peak intensities defined as $I=\sqrt{I_{1} I_{2}}$ of $2.5 \times 10^{14} \mathrm{~W} \mathrm{~m}^{-2}(3.9 \mathrm{~K})$ to $4.0 \times 10^{15} \mathrm{~W} \mathrm{~m}^{-2}(62 \mathrm{~K})$. Figure 3 shows recorded spectra at the two extremes of this intensity range, as well as the calculated CRS spectral profile determined from measurements of laser intensity, pulse shape, and spatial profile. The relative intensity of the CRS scattered light for these two extremes differs by approximately 3 orders of magnitude and thus for comparison of the two spectral profiles we normalise the profile for each case to the peak value at zero detuning for that intensity. For the low intensity case shown in Fig. 3 ( $2.5 \times$ $10^{14} \mathrm{~W} \mathrm{~m}^{-2}$ ), the line shape is well approximated by the near Gaussian profile as predicted from conventional coherent Rayleigh scattering also shown in this figure. In contrast, the high intensity spectral profile shows the expected narrowing near line center and also the occurrence

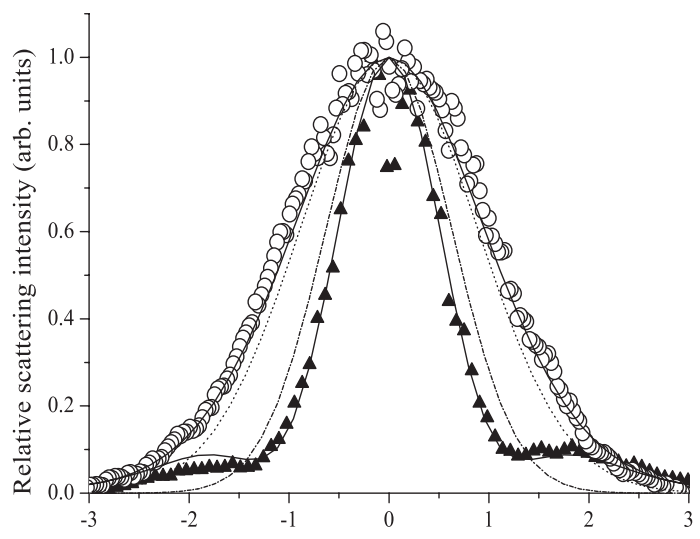

FIG. 3. A comparison between coherent Rayleigh scattering spectral profile from $\mathrm{CO}_{2}$ gas at two pump intensities of $2.5 \times$ $10^{14}$ (circles) and $4 \times 10^{15} \mathrm{~W} \mathrm{~m}^{-2}$ (triangles). Spectral narrowing to approximately half the width at low intensity is observed and is in good agreement with the predicted line shape (solid lines). Also shown for reference is the Doppler profile from spontaneous Rayleigh (dashed line) and a profile that is proportional to the square of the original distribution function (dotdashed line). 


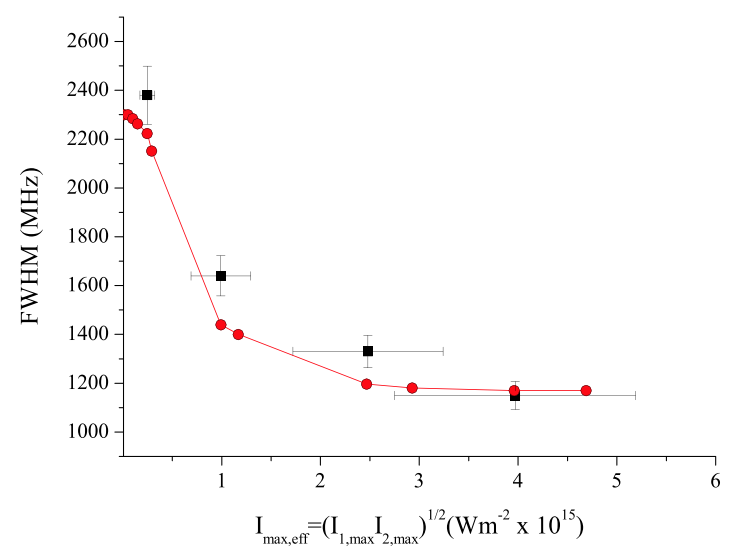

FIG. 4 (color online). The full width half maximum of the coherent Rayleigh scattering line shape with increasing pump intensity. The squares, with error bars, are the experimental points and the circles are the calculated values.

of wide pedestals in the wings that coincide with the low intensity CRS profile at the largest pump laser detunings. Both of these effects agree generally with our expectations from Fig. 2(c) and also with the simulations of utilizing the parameters of the optical fields in the experiment. The slight asymmetry in the higher power CRS spectrum was found to originate from a phase mismatch due to a beam pointing instability in one of our lasers [18]. Also plotted on this graph is the square of the original distribution function which was found to approximate the CRS signal near line center, but not in the wings where few of the molecules are trapped by the potential. The variation in line shape and the expected saturation of the narrowing with pump intensity is more clearly shown over the intensity range that we considered when we plot the full width half maximum (FWHM) of the CRS spectrum as a function of pump beam intensity as shown in Fig. 4. The measured widths are indicated by the squares and the red points are the calculated spectral widths over a wider intensity range than we used in our experiments. This figure shows clearly the narrowing of line shape by a factor of approximately 2 with an order of magnitude change in the pump intensity, and agrees well with the calculated profiles.

We have found that as the pressure of the gas is increased to the point where the mean collision time is shorter than the pulse duration, collisions lead to loss of molecules from the trapped region. This process has the same effect as reducing the well depth of the potential and thus scattering becomes dominated by untrapped particles. The line shape for this case was found to broaden to approximately the width observed in the collisionless regime at low intensity in Figs. 3 and 4 and this was observed with increasing pressure from 10 to 200 mbar.

In conclusion, we have observed a new type of spectral narrowing and saturation of the CRS signal which we attribute to the increased localization of molecules near and within the optical potential induced by the two pump beams. The narrowing is a spectral signature that the potential is large enough to trap a significant fraction of the distribution function even for the brief $10 \mathrm{~ns}$ duration of the applied optical fields. The narrowing of the CRS spectrum has important implications for the accuracy of diagnostic applications of this technique [19] when high intensities are used because within a relatively small intensity range the spectral line shape can change significantly. It should also be apparent in Bragg scattered spectra from ultracold atoms trapped when using the coherent Rayleigh scattering as described here. The narrowed CRS line shape observed in our experiments is not Dicke narrowing since the scattered signal intensity is proportional to the total induced density perturbation at each pump beam frequency difference and not the total spectral profile of the scattered light. Dicke narrowing should however also be observed in the spectrally resolved Rayleigh scattering along the axis of the pump beams and in resonant absorption processes induced by a third field. This feature may be a useful means for performing nonresonant, near Doppler free measurements in room temperature gases.

[1] R. H. Dicke, Phys. Rev. 89, 472 (1953).

[2] R. L. Mössbauer, Z. Phys. 151, 124 (1958).

[3] R. Blackmore, S. Green, and L. Monchick, J. Chem. Phys. 91, 3846 (1989).

[4] J.C. Berguist, W. M. Itano, and D. J. Wineland, Phys. Rev. A 36, 428 (1987).

[5] C. I. Westbrook et al., Phys. Rev. Lett. 65, 33 (1990).

[6] G. Grynberg, B. Lounis, P. Verkerk, J.-Y. Courtois, and C. Salomon, Phys. Rev. Lett. 70, 2249 (1993).

[7] G. Birkl, M. Gatzke, I.H. Deutsch, S. L. Rolston, and W. D. Phillips, Phys. Rev. Lett. 75, 2823 (1995).

[8] I. H. Deutsch, R. J. C. Spreeuw, S. L. Rolston, and W. D. Phillips, Phys. Rev. A 52, 1394 (1995).

[9] S. Slama, C. von Cube, B. Deh, A. Ludewig, C. Zimmermann, and $\mathrm{Ph}$. W. Courteille, Phys. Rev. Lett. 94, 193901 (2005).

[10] J. H. Grinstead and P. F. Barker, Phys. Rev. Lett. 85, 1222 (2000).

[11] E. B. Cummings, Opt. Lett. 19, 1361 (1994).

[12] A. Stampanoni-Panariello, D. N. Kozlov, P. P. Radi, and B. Hemmerling, Appl. Phys. B 81, 101 (2005).

[13] P. F. Barker and M. N. Shneider, Phys. Rev. A 64, 033408 (2001).

[14] X. Pan et al., Opt. Lett. 27, 161 (2002).

[15] H. T. Bookey, A. I. Bishop, and P. F. Barker, Opt. Express 14, 3461 (2006).

[16] X. Pan, M. N. Shneider, and R. B. Miles, Phys. Rev. Lett. 89, 183001 (2002).

[17] M. N. Shneider, P. F. Barker, X. Pan, and R. B. Miles, Opt. Commun. 239, 205 (2004).

[18] H. T. Bookey, A. I. Bishop, M. N. Shneider, and P. F. Barker, J. Raman Spectrosc. 37, 655 (2006).

[19] W. Marques, Jr., J. Stat. Mech. (2007) P03013. 